\title{
163. Effects of Red and Far-Red Light upon the Yellow Pigment of Excised Secondary Root Apices of Phaseolus aureus*)
}

\author{
By Hideo ToRIYAMA ${ }^{* *}$ and Mordecai J. JAFFE***) \\ (Comm. by Tetsu Sakamura, M. J. A., Nov. 12, 1973)
}

In 1968, Tanada ${ }^{3), 4)}$ had investigated the electrotropic response of root tips of barley and mung bean, mediated by phytochrome. Tanada, ${ }^{3), 4)}$ Jaffe $^{1)}$ and Younghans and $\mathrm{Jaffe}^{6}$ ) found that the excised root tips from barley and mung bean seedlings become attached to a glass surface negatively charged, when irradiated with red light, while they were released from it by the successive irradiations with far-red light.

During the studies on the stimulus response of mung bean roots, the present authors noted that a native pigmented area could be seen with the naked eye at the apex of each secondary root. By employing histological and cytological techniques the writers were able to observe a migration of yellow pigment and a remarkable behaviour of the protoplast in the sieve tube, caused by the irradiation with red light and far-red light.

Materials and methods. Young mung bean (Phaseolus aureus) seedlings were grown under continuous illumination as previously described, ${ }^{6)}$ and those of 12-14 days old were used for irradiation and subsequent observation. The red and far-red light irradiations were performed in the same way as discribed by Yunghans and Jaffe, ${ }^{6)}$ under the following regimes of $a$ ) dark, $b$ ) dark and red, $c$ ) dark and red and far-red. The duration of every dark and irradiation equally counted four minutes.

The distal 5-8 $\mathrm{mm}$ root tips excised from the secondary root of 10-25 mm length were submerged in shallow distilled water contained in a petri dish. Immediately after the irradiation, the materials were fixed fixatives. For observation by the naked eye, and with the photomicrographs, Müller's fluid (distilled water $10 \mathrm{ml} ; \mathrm{K}_{2} \mathrm{Cr}_{2} \mathrm{O}_{7}, 2.5 \mathrm{~g}$; $\mathrm{Na}_{2} \mathrm{SO}_{4}, 1 \mathrm{~g}$ ) was employed. After the completion of fixation, these tips were thoroughly washed in running water for 5 hours to remove to M. J. Jaffe.

*) Supported by National Science Foundation (U. S. A.) grant \#BG 33257

\footnotetext{
**) Department of Biology, Tokyo Women's Christian College, Tokyo 167, Japan.

***) Department of Botany, Ohio University, Athens, Ohio 45701, U. S. A.
} 
traces of the fixing reagents. The materials were kept in 5\% tertiary butyl alcohol, embedded in pure glycerin on the glass slide and then covered with a cover slip. The non-sectioned and non-stained materials thus prepared, were observed with the naked eye or by using a microscope.

In order to measure the dimension of the sieve tube in variously irradiated root tips, the materials were fixed with Lillie's neutral buffered formalin ${ }^{2}$ (commercial formalin, $100 \mathrm{ml}$; distilled water, $900 \mathrm{ml} ; \mathrm{NaH}_{2} \mathrm{PO}_{4} \cdot \mathrm{H}_{2} \mathrm{O}, 0.4 \mathrm{~g} ; \mathrm{Na}_{2} \mathrm{HPO}_{4} 6.5 \mathrm{~g}$ ). After 10 hour's fixation, they were thoroughly washed in running water for several hours to remove any trace of fixatives. Water was replaced with a dehydration series of tertiary butyl alcohol, and the materials were embedded in paraffin. Paraffin sections were prepared 15-20 micra thick. The sections were then stained with $1 \%$ alizarin red sulfonate, as previously described by Toriyama and Jaffe..$^{5)}$ It is noted that, the section of the material which had been irradiated with far-red light, had a tendency to peel off the glass slide during the staining process. In that case, the preparation was soaked in a $0.5 \%$ celloidin solution (celloidin, $0.5 \mathrm{~g}$; ethanol $50 \mathrm{ml}$; ethyl ether, $50 \mathrm{ml}$ ) before the staining.

Results. The yellow pigment in the secondary root tip of mung

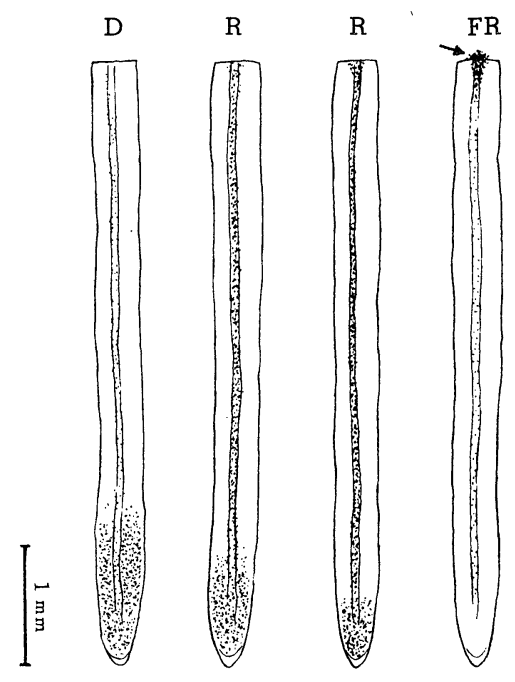

Fig. 1. Schematic figure of excised root tip of Phaseolus aureus secondary root. The materials were fixed with Müller's fluid and mounted in glycerin. The dotted area indicates the distribution of yellow pigment. D: $4 \mathrm{~min}$. dark, $\mathrm{R}$ : 4 min. dark +4 min. red, FR: 4 min. dark +4 min. red +4 min. far-red. Arrow indicates yellow pigment on the cut end of excised root tip. 
[Vol. 49,

bean seedling showed a change of localization, according to the irradiation red and/or far-red light. Example of the results is shown in Fig. 1. There were the obvious localization changes of the yellow pigment at the site of the vascular bundle and in the distal $1 \mathrm{~mm}$ of the root tips. In the material which was kept in the dark, the yellow pigment appeared continuously in the distal part of the root tip (Fig. 1D, 2a3). A small amount of yellow pigment could be seen

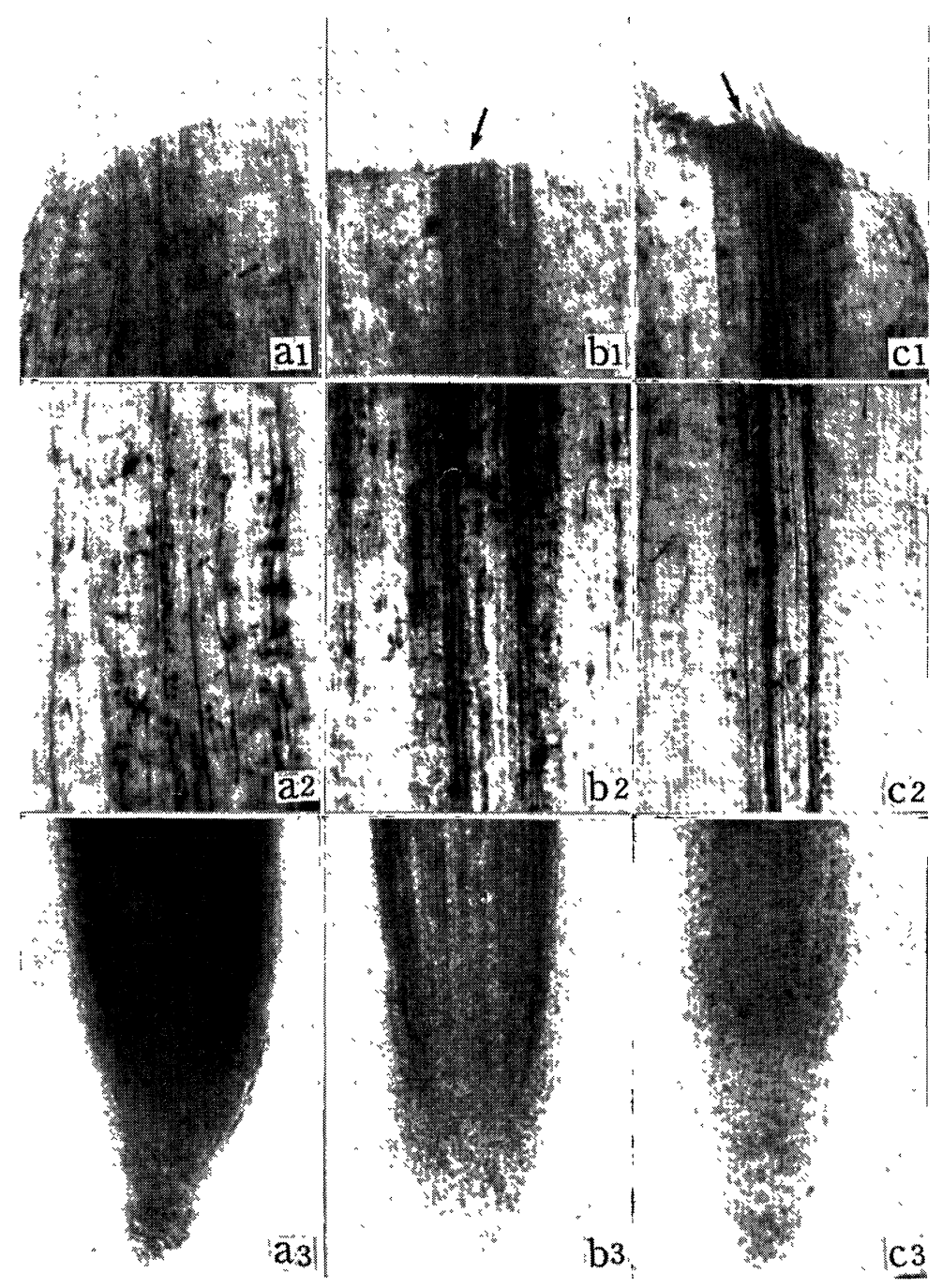

Fig. 2. The migration of yellow pigment in excised root tips of Phaseolus aureus. The materials were fixed with Müller's fluid and mounted in glycerin. $\times 33$. a1, a2, a3: 4 min. dark; b1, b2, b3: 4 min. dark +4 min. red, c1, c2, c3: 4 min. dark +4 min. red +4 min. far-red. Arrows indicate yellow pigment on the cut end of vascular bundle. 
also in the vascular bundles. Those tissues containing the pigment can be identified with sieve tubes, shown in Fig. 3a indicated with the arrow. It was ascertained that there was no yellow pigment in the cut end of these tissues (Figs. 1D, 2a1).

In the material of irradiation $b$ (dark and red), it was found that the yellow pigment decreased in the distal part of the root tip. Under these experimental conditions, the yellow pigment can be seen also in the vascular bundle (Figs. 1R, 2b2). These tissues can be identified with sieve tube, as shown in Figs. 3b1, b2. The yellow pigment appeared also in the vascular bundle, probably in sieve tubes, near the cut end of the tissue as indicated with arrow (Fig. 2b1).

In the material of irradiation $c$ (dark and red and far-red) the yellow pigment decreased in the distal part of root tip (Figs. 1FR, 2c3) and appeared in the thin protoplast of the sieve tube (Figs. 2c2, $3 c)$. At the cut end of the tissue a lot of yellow pigment was found as indicated with arrow (Figs. 1FR, 2c1).

The yellow pigment in the protoplast of the sieve tube was stained in orange color with alizarin red sulfonate. These materials are convenient to use for microscopical observation. The diameter of the protoplast of the sieve tubes was measured at their linear center, and sieve tube of the red light irradiated roots were found to be considerably thicker than those of roots kept dark or irradiated with farred light (Fig. 4). The end of the protoplast of the sieve tube in the preparation of red light irradiation became separated from the thin cell wall (Figs. 3b1, b2). Probably, the protoplast of the sieve tube may contract in the longitudinal direction, due to the red light irradia-

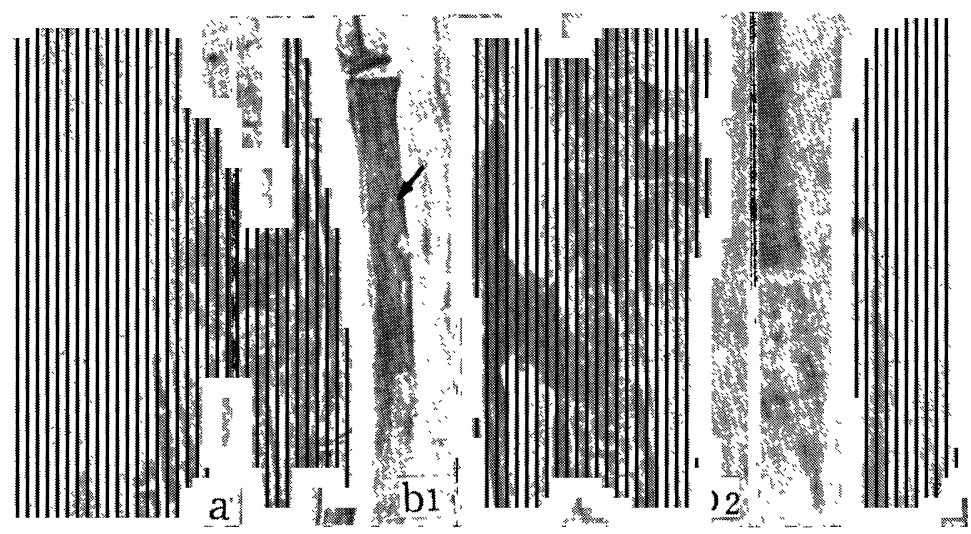

Fig. 3. Behaviour of the protoplast, which retains the yellow pigment in the sieve tube of Phaseolus aureus. The materials were fixed with Lillie's neutral buffered formalin and stained with alizarin red S. $\times 500$. a: 4 min. dark; b1, b2: 4 min. dark +4 min. red; c: 4 min. dark +4 min. red +4 min. far-red. Arrows indicate the protoplast of the sieve tube. 


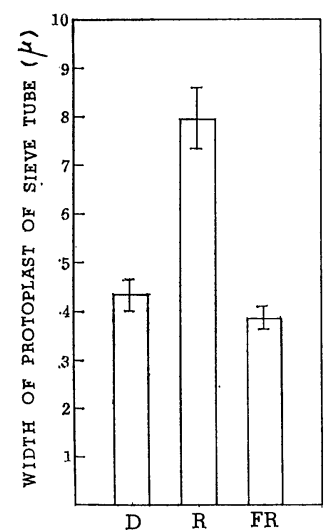

Fig. 4. Variation of the width of the protoplast in sieve tube of Phaseolus aureus secondary root tip. The materials were fixed with Lillie's neutral bufferd formalin and stained with alizarin red $\mathrm{S}$. $\mathrm{D}: 4 \mathrm{~min}$. dark; $\mathrm{R}$ : $4 \mathrm{~min}$. dark $+4 \mathrm{~min}$. red; FR: 4 min. dark +4 min. red +4 min. far-red.

tion. It may be added here that the protoplast of the sieve tube of the roots irradiated with far-red light, showed the same feature of those kept dark for four minutes.

Discussion. The experiments described in the present paper indicate that the endogenous yellow pigment which is normally located in the apical region of secondary roots of mung bean, migrated upward due to the influence of irradiation with red light. But its original localization could not be recognized after an irradiation with far-red light. This observation may suggest us what happened in the behaviour of the yellow pigment during the far-red irradiation. In this relation, we should note also that the yellow pigment was observable in the cut end of the excised roots (Figs. 1FR 2c1). Probably, the yellow pigment went out from the sieve tubes at the cut end of the tissue and was diffused into the medium solution or distilled water, during the irradiation with far-red light. At any rate, it seems to us, the histological response due to red and/or far-red light may be mediated by phytochrome. The relationship between the behaviour of the protoplast of the sieve element and the migration of yellow pigment, is a worthwhile subject for future investigation. The result of further studies on the role of the sieve element in an intact root, under the red and far-red irradiation, will be reported in another paper in the near future.

The main part of the work reported here was done at Department of Botany, Ohio University, Athens, Ohio, U. S. A., from Dec. 1969 to Oct. 1970. 


\section{References}

1) Jaffe, M. J.: Science, 162, 1016-1017 (1968).

2) Lillie, R. D.: Histopathologic Technique. Philadelphia, Blakiston (1948).

3) Tanada, T.: Proc. Natl. Acod. Sci. U. S., 49, 376-380 (1968).

4) —-: Plant Physiol., 43, 2070-2071 (1968).

5) Toriyama, H., and M. J. Jaffe: Plant Physiol., 49, 71-81 (1972).

6) Yunghans, H., and M. J. Jaffe: Physiol. Planta., 23, 1004-1016 (1970). 